Research Article

\title{
On a Discrete Markov-Modulated Risk Model with Random Premium Income and Delayed Claims
}

\author{
Changwei Nie, ${ }^{1}$ Mi Chen, ${ }^{1,2}$ and Haiyan Liu $\mathbb{D}^{1,2}$ \\ ${ }^{1}$ College of Mathematics and Informatics, Fujian Normal University, Fuzhou 350117, China \\ ${ }^{2}$ Fujian Provincial Key Laboratory of Mathematical Analysis and Its Applications, Fuzhou 350117, China \\ Correspondence should be addressed to Haiyan Liu; rain6397@163.com
}

Received 26 March 2020; Accepted 10 August 2020; Published 20 October 2020

Guest Editor: Wenguang Yu

Copyright (C) 2020 Changwei Nie et al. This is an open access article distributed under the Creative Commons Attribution License, which permits unrestricted use, distribution, and reproduction in any medium, provided the original work is properly cited.

In this paper, a discrete Markov-modulated risk model with delayed claims, random premium income, and a constant dividend barrier is proposed. It is assumed that the random premium income and individual claims are affected by a Markov chain with finite state space. The model proposed is an extension of the discrete semi-Markov risk model with random premium income and delayed claims. Explicit expressions for the total expected discounted dividends until ruin are obtained by the method of generating function and the theory of difference equations. Finally, the effect of related parameters on the total expected discounted dividends are shown in several numerical examples.

\section{Introduction}

The study of dividend strategies for the insurance risk model was first proposed by De Finetti [1], and he found that the optimal strategy must be a barrier strategy in the studied discrete time model. Since then, many scholars have tried to work out the dividend problems under more general and more realistic model assumptions, for example, Claramunt et al. [2], Zhou [3], Landriault [4], Gerber et al. [5], Chen et al. [6], Chen and Yuen [7], and Peng et al. [8, 9].

In practice, insurance claims may be delayed for various reasons. Yuen and Guo [10] considered a reasonable claim structure that includes two types of independent claims, namely, main claims and by-claims, but the occurrence of by-claims may be delayed. For example, for a catastrophe such as an earthquake or a rainstorm, it is very likely that there exist other insurance claims after the immediate ones. From then on, the related issues about a risk process with such time-correlated claims have been studied by many authors. $\mathrm{Li}$ and $\mathrm{Wu}$ [11] calculated the total expected discounted dividends up to the time of ruin in a discrete time risk model with delayed claims and a constant dividend barrier. Other risk models involving delayed claims were studied by Xiao and Guo [12], Bao and Liu [13], Zhou et al.
[14], Yuen et al. [15], Liu and Zhang [16], Deng et al. [17], and the references therein.

As an extension of the classical risk model, the risk model with random premium income has attracted a great deal of attention during the last few years. Boikov [18] generalized the classical risk model to the case where the premium process is determined by a compound Poisson process. Bao [19] extended the classical risk model to the case where the premium income process is a Poisson process and studied the expected discounted penalty at ruin. Assuming that the aggregate premium process is modeled as a compound Poisson process, Zou et al. [20] studied the expected discounted penalty function and optimal dividend strategy for a risk model with interclaim-dependent claim sizes. For the risk models with random premium income, one can also see [21-24] for more details.

Markov-modulated risk model where the surplus process is affected by an environmental Markov chain is a quite important model which attributed to the structural changes in conditions of economic, politic, or social, etc. Zhu and Yang [25] considered some ruin problems in a Markov regime-switching risk model where dividends were paid out according to a certain threshold strategy depending on the underlying Markovian environment process. Chen et al. [26] 
studied the survival probability for a discrete semi-Markov risk model, which assumes individual claims are influenced by a Markov chain with finite state space. Yuen et al. [27] investigated the expected penalty functions for a discrete semi-Markov risk model with randomized dividends. Other related works can be found in $\mathrm{Lu}$ and $\mathrm{Li}$ [28], Diko and Usábel [29], Chen et al. [30], Huo et al. [31], etc.

In the discrete time risk model with random premium income, the authors often assumed that the random premium income follows a binomial process with a certain parameter, which means that the premium received in each period is a sequence of independent variables with the same Bernoulli distribution. In this paper, we propose a discrete time risk model where the random premium process and the individual claims are all influenced by a Markov chain. The model proposed in this paper is an extension of the discrete semi-Markov risk model with paying dividends and delayed claims. As mentioned in Chen et al. [26], the discrete semiMarkov risk model includes several existing risk models such as the compound binomial model and the compound Markov binomial model as special cases. So the discrete model studied in this paper is quite general. The explicit expressions for the expected discounted dividends until ruin are obtained for the model.

The outline of this paper is as follows. In Section 2, we introduce the model of this paper, including various parameters and notations. In Section 3, explicit expressions for the total expected discounted dividends until ruin are obtained by the method of generating function and the theory of difference equations. In Section 4, numerical examples are provided to illustrate the impact of the related parameters on the total expected discounted dividends.

\section{Problem Formulation}

We first recall the discrete time risk model with delayed claims that are studied by Yuen and Guo [10]. Denote the discrete time period by $t=0,1,2, \ldots$, and assume that premiums are received with a constant premium rate of 1 in each time period. The probability of a main claim occurring in any time period is $p(0<p<1)$, and the probability of no main claim is $q=1-p$. Each main claim causes a by-claim, the probability that the main claim and by-claim occurs in the same period is $\theta$, or the occurrence of by-claim is delayed to next time period with probability $1-\theta$. Suppose that $\left\{\xi_{k}, k=1,2, \ldots\right\}$ be a sequence of Bernoulli random variables with $\mathbb{P}\left(\xi_{k}=1\right)=p$ and $\mathbb{P}\left(\xi_{k}=0\right)=1-p$, describing whether or not a main claim occurs in the $k$-th time period, and $\left\{\eta_{k}, k=1,2, \ldots\right\}$ be another sequence of Bernoulli random variables with $\mathbb{P}\left(\eta_{k}=1\right)=\theta$ and $\mathbb{P}\left(\eta_{k}=0\right)=1-\theta$, describing whether or not the $k$-th by-claim occurs simultaneously with its corresponding main claim. Let $\left\{X_{k}, k=1,2, \ldots\right\}$ be the independent and identically distributed (i.i.d.) main claims amounts and $\left\{Y_{k}, k=1,2, \ldots\right\}$ be the independent and identically distributed (i.i.d.) byclaims amounts. Then the total amount of the main claims and by-claims until the end of $t$-th time period are given by

$$
\begin{aligned}
S_{t}^{X} & =\sum_{k=1}^{t} \xi_{k} X_{k}, \quad t=1,2, \ldots, \\
S_{1}^{Y} & =\xi_{1} \eta_{1} Y_{1}, \\
S_{t}^{Y} & =\sum_{k=1}^{t} \xi_{k} \eta_{k} Y_{k}+\sum_{k=2}^{t} \xi_{k-1}\left(1-\eta_{k-1}\right) Y_{k-1}, \quad t=2,3, \ldots
\end{aligned}
$$

Hence, the surplus process for an insurance company is described as follows:

$$
S(t)=u+t-S_{t}^{X}-S_{t}^{Y}
$$

where $u$ is the initial surplus of the insurance company.

Now we introduce the risk model with delayed claims, a constant dividend barrier, and random premium income. We assume that premium income and dividends occur at the beginning of the period, and each claim occurs at the end of the period. If the surplus is above a constant barrier $b$, then the excess is paid out as a dividend. In addition, if the initial surplus $u>b, u-b$ is paid out as a dividend immediately. Denote by $Z_{k}$ the amount of premium received in the $k$-th time period. Then the surplus process at the end of $t$-th time period of the risk model with delayed claims, a constant dividend barrier, and random premium income can be expressed as

$$
U(t)=u+\sum_{k=1}^{t} Z_{k}-S_{t}^{X}-S_{t}^{Y}-\sum_{k=0}^{t} d_{k},
$$

where $d_{k}$ is the amount of dividend paid out in the $k$-th period with $d_{0}=\max \{u-b, 0\}$ and $\sum_{k=1}^{0} Z_{k}=0$.

In this paper, we assume that $\left\{J_{k}, k=0,1, \ldots\right\}$ is a Markov chain with the state space $M=\{1, \ldots, r\}$, and its transition matrix is written as $\mathbf{P}=\left(p_{i j}\right)_{i, j \in M}$, where $p_{i j}=\mathbb{P}\left(J_{k+1}=j \mid J_{k}=i, J_{t}, t \leq k-1\right)$. Furthermore, the distributions of premium income, main claims, and by-claims that depend on the Markov chain are defined as follows:

$$
\begin{aligned}
& q_{i j}(l)=\mathbb{P}\left(Z_{t}=l \mid J_{t}=j, J_{t-1}=i, J_{k}, Z_{k}, k \leq t-1\right), \quad l=0,1, \\
& g_{i j}(n)=\mathbb{P}\left(X_{t}=n \mid J_{t}=j, J_{t-1}=i, J_{k}, X_{k}, k \leq t-1\right), \quad n=1,2, \ldots, \\
& f_{i j}(m)=\mathbb{P}\left(Y_{t}=m \mid J_{t}=j, J_{t-1}=i, J_{k}, Y_{k}, k \leq t-1\right), \quad m=1,2, \ldots
\end{aligned}
$$

For simplicity, this article only considers the case of $M=\{1,2\}$. In addition, we agree that $\sum_{i=1}^{2} \sum_{j=1}^{2} q_{i j}(1) \neq 0$; otherwise, there must be no premium income in any period. Define $T_{u, b}=\inf \{k \geq 1: U(k)<0\}$ to be the ruin time of 
model (3) and the one-period discount factor is $0<v \leq 1$. Then the total expected discounted dividends until ruin given the initial surplus $u$ and initial state $i$ are defined as follows:

$$
V_{i}(u, b)=E\left[\sum_{k=0}^{T_{u, b}} v^{k} d_{k} \mid U(0)=u, J_{0}=i\right], \quad i=1,2 .
$$

The rest of this paper is devoted to obtain the explicit expressions of $V_{i}(u, b)$ for calculation purposes.

\section{The Total Expected Discounted \\ Dividends $V_{i}(u, b)$}

In order to obtain the explicit expressions of the total expected discounted dividends, we need to consider the scenario that the by-claim induced by the main claim is delayed to the next period (see Yuen and Guo [10]). According to this scenario, we define a complementary surplus process as follows:

$$
U_{1}(t)=u+\sum_{k=1}^{t} Z_{k}-S_{t}^{X}-S_{t}^{Y}-\sum_{k=0}^{t} d_{k}-Y I_{(t \geq 1)}
$$

where $I_{A}$ is the indicator function of event $A, Y$ is a random variable following the same probability function with $\left\{Y_{k}, k=1,2, \ldots\right\}$ and is independent of all other claim amounts random variables $X_{i}$ and $Y_{j}$ for all $i$ and $j$. The total expected discounted dividends of the complementary surplus process until ruin are denoted by $V_{1, i}(u, b)$.

Based on the condition of premium income, claims, and dividends, the following equations can be obtained by using the law of total probability for the first period.

For $u=0,1, \ldots, b$,

$$
\begin{aligned}
V_{i}(u, b)= & v q \sum_{j=1}^{2} p_{i j} q_{i j}(0) V_{j}(u, b)+v p \theta \sum_{j=1}^{2} \sum_{m+n \leq u} p_{i j} q_{i j}(0) g_{i j}(m) f_{i j}(n) V_{j}(u-m-n, b) \\
& +v q \sum_{j=1}^{2} p_{i j} q_{i j}(1) V_{j}(u+1, b)+v p \theta \sum_{j=1}^{2} \sum_{m+n \leq u+1} p_{i j} q_{i j}(1) g_{i j}(m) f_{i j}(n) V_{j}(u+1-m-n, b) \\
& +v p(1-\theta) \sum_{j=1}^{2} \sum_{m=1}^{u} p_{i j} q_{i j}(0) g_{i j}(m) V_{1, j}(u-m, b) \\
& +v p(1-\theta) \sum_{j=1}^{2} \sum_{m=1}^{u+1} p_{i j} q_{i j}(1) g_{i j}(m) V_{1, j}(u+1-m, b), \quad i=1,2 \\
V_{1, i}(0, b)= & v q \sum_{j=1}^{2} p_{i j} q_{i j}(1) f_{i j}(1) V_{j}(0, b), \quad i=1,2
\end{aligned}
$$

and for $u=1, \ldots, b$,

$$
\begin{aligned}
V_{1, i}(u, b)= & v q \sum_{j=1}^{2} \sum_{l=1}^{u} p_{i j} q_{i j}(0) f_{i j}(l) V_{j}(u-l, b)+v q \sum_{j=1}^{2} \sum_{l=1}^{u+1} p_{i j} q_{i j}(1) f_{i j}(l) V_{j}(u+1-l, b) \\
& +v p \theta \sum_{j=1}^{2} \sum_{m+n+l \leq u} p_{i j} q_{i j}(0) g_{i j}(m) f_{i j}(n) f_{i j}(l) V_{j}(u-m-n-l, b) \\
& +v p \theta \sum_{j=1}^{2} \sum_{m+n+l \leq u+1} p_{i j} q_{i j}(1) g_{i j}(m) f_{i j}(n) f_{i j}(l) V_{j}(u+1-m-n-l, b) \\
& +v p(1-\theta) \sum_{j=1}^{2} \sum_{m+l \leq u} p_{i j} q_{i j}(0) g_{i j}(m) f_{i j}(l) V_{1, j}(u-m-l, b) \\
& +v p(1-\theta) \sum_{j=1}^{2} \sum_{m+l \leq u+1} p_{i j} q_{i j}(1) g_{i j}(m) f_{i j}(l) V_{1, j}(u+1-m-l, b), \quad i=1,2 .
\end{aligned}
$$


According to the barrier dividend strategy, for $u=b+1, \ldots$, we have

$$
\begin{gathered}
V_{i}(u, b)=u-b+V_{i}(b, b), \quad i=1,2, \\
V_{1, i}(u, b)=u-b+V_{1, i}(b, b), \quad i=1,2 .
\end{gathered}
$$

From (7) and (9), $V_{1, i}(u, b)$ can be rewritten as

$$
V_{1, i}(u, b)=\sum_{l=1}^{u} V_{i}(u-l, b) \sum_{j=1}^{2} p_{i j} f_{i j}(l), \quad i=1,2 .
$$

This result can also be obtained from model (6) as

$$
\begin{aligned}
V_{1, i}(u, b) & =E\left[V_{i}(u-Y, b)\right] \\
& =\sum_{l=1}^{u} V_{i}(u-l, b) \sum_{j=1}^{2} p_{i j} f_{i j}(l), \quad i=1,2 .
\end{aligned}
$$

Substituting (8) into (7), we get

$$
\begin{aligned}
V_{i}(0, b)= & v q \sum_{j=1}^{2} p_{i j} q_{i j}(0) V_{j}(0, b) \\
& +v q \sum_{j=1}^{2} p_{i j} q_{i j}(1) V_{j}(1, b) \\
& +v^{2} p q(1-\theta) \sum_{j=1}^{2} p_{i j} q_{i j}(1) g_{i j}(1) \\
\sum_{k=1}^{2} p_{j k} q_{j k}(1) f_{j k} & \cdot(1) V_{k}(0, b), \quad i=1,2 .
\end{aligned}
$$

Similarly, substituting (11) into (7), we have

$$
\begin{aligned}
V_{i}(u, b)= & v q \sum_{j=1}^{2} p_{i j} q_{i j}(0) V_{j}(u, b) \\
& +v q \sum_{j=1}^{2} p_{i j} q_{i j}(1) V_{j}(u+1, b) \\
& +v p \sum_{j=1}^{2} \sum_{m+n \leq u} p_{i j} h_{i j}^{X}(m) V_{j}(u-m-n, b) \\
& \cdot\left[\theta f_{i j}(n)+(1-\theta) \sum_{k=1}^{2} p_{j k} f_{j k}(n)\right], \\
u= & 1,2, \ldots, b, i=1,2,
\end{aligned}
$$

where $\quad h_{i j}^{X}(m)=q_{i j}(0) g_{i j}(m)+q_{i j}(1) g_{i j}(m+1) \quad$ with $g_{i j}(0)=0, m=0,1,2, \ldots$.

To obtain the explicit expressions of $V_{i}(u, b)$, we define new functions $W_{i}(u)$ as follows:

$$
\begin{aligned}
W_{i}(0)= & v q \sum_{j=1}^{2} p_{i j} q_{i j}(0) W_{j}(0)+v q \sum_{j=1}^{2} p_{i j} q_{i j}(1) W_{j}(1) \\
& +v^{2} p q(1-\theta) \sum_{j=1}^{2} p_{i j} q_{i j}(1) g_{i j}(1) \sum_{k=1}^{2} p_{j k} q_{j k}(1) f_{j k} \\
& \cdot(1) W_{k}(0), \quad i=1,2 .
\end{aligned}
$$

$$
\begin{aligned}
& W_{i}(u)= v q \sum_{j=1}^{2} p_{i j} q_{i j}(0) W_{j}(u)+v q \sum_{j=1}^{2} p_{i j} q_{i j}(1) W_{j}(u+1) \\
&+v p \sum_{j=1}^{2} \sum_{m+n \leq u} p_{i j} h_{i j}^{X}(m) W_{j}(u-m-n) \\
& \cdot\left[\theta f_{i j}(n)+(1-\theta) \sum_{k=1}^{2} p_{j k} f_{j k}(n)\right], \\
& u \in \mathbb{N}_{+}, i=1,2 .
\end{aligned}
$$

We use $\widetilde{h}_{i j}^{X}(s), \widetilde{f}_{i j}(s)$, and $\widetilde{W}_{i}(s)$ to represent the generating functions of $h_{i j}^{X}(k), f_{i j}(k)$, and $W_{i}(k)$, respectively. Multiplying both sides of (15) and (16) by $s^{u+1}$, next summing over $u$ from 0 to $\infty$ gives

$$
\begin{aligned}
\frac{s}{v} \widetilde{W}_{i}(s)= & s q \sum_{j=1}^{2} p_{i j} q_{i j}(0) \tilde{W}_{j}(s)+q \sum_{j=1}^{2} p_{i j} q_{i j}(1)\left(\tilde{W}_{j}(s)-W_{j}(0)\right) \\
& +s p \sum_{j=1}^{2} p_{i j} \widetilde{W}_{j}(s) \tilde{h}_{i j}^{X}(s)\left[\theta \widetilde{f}_{i j}(s)+(1-\theta) \sum_{k=1}^{2} p_{j k} \tilde{f}_{j k}(s)\right] \\
& +\operatorname{svpq}(1-\theta) \sum_{j=1}^{2} p_{i j} q_{i j}(1) g_{i j}(1) \sum_{k=1}^{2} p_{j k} q_{j k}(1) f_{j k}(1) W_{k}(0), \quad i=1,2 .
\end{aligned}
$$


For the convenience of the following derivations, we define

$$
\begin{aligned}
& \tilde{e}_{1}(s)=q \sum_{j=1}^{2} p_{1 j} q_{1 j}(1) W_{j}(0)-s v p q(1-\theta) \sum_{j=1}^{2} p_{1 j} q_{1 j}(1) g_{1 j}(1) \sum_{k=1}^{2} p_{j k} q_{j k}(1) f_{j k}(1) W_{k}(0), \\
& \widetilde{e}_{2}(s)=q \sum_{j=1}^{2} p_{2 j} q_{2 j}(1) W_{j}(0)-s v p q(1-\theta) \sum_{j=1}^{2} p_{2 j} q_{2 j}(1) g_{2 j}(1) \sum_{k=1}^{2} p_{j k} q_{j k}(1) f_{j k}(1) W_{k}(0) \text {, } \\
& \tilde{e}_{i j}(s)=q p_{i j} q_{i j}(1)+s\left[q p_{i j} q_{i j}(0)+p p_{i j} \tilde{h}_{i j}^{X}(s)\left(\theta \tilde{f}_{i j}(s)+(1-\theta) \sum_{k=1}^{2} p_{j k} \tilde{f}_{j k}(s)\right)\right], \quad i, j=1,2 . \\
& {\left[\left(\widetilde{e}_{11}(s)-\frac{s}{v}\right)\left(\tilde{e}_{22}(s)-\frac{s}{v}\right)-\tilde{e}_{12}(s) \widetilde{e}_{21}(s)\right] \widetilde{W}_{1}(s)} \\
& =\widetilde{e}_{1}(s)\left(\widetilde{e}_{22}(s)-\frac{s}{v}\right)-\widetilde{e}_{2}(s) \widetilde{e}_{12}(s) .
\end{aligned}
$$

$$
\left\{\begin{array}{l}
\left(\tilde{e}_{11}(s)-\frac{s}{v}\right) \widetilde{W}_{1}(s)+\widetilde{e}_{12}(s) \widetilde{W}_{2}(s)=\widetilde{e}_{1}(s), \\
\tilde{e}_{21}(s) \widetilde{W}_{1}(s)+\left(\tilde{e}_{22}(s)-\frac{s}{v}\right) \widetilde{W}_{2}(s)=\widetilde{e}_{2}(s) .
\end{array}\right.
$$

Simplifying the above equation yields

$$
\begin{aligned}
a_{i}(0) & =q p_{i i} q_{i i}(1), \\
a_{i}(1) & =q p_{i i} q_{i i}(0)+p p_{i i} h_{i i}^{X}(0)\left[\theta f_{i i}(0)+(1-\theta) \sum_{k=1}^{2} p_{i k} f_{i k}(0)\right]-\frac{1}{v}, \\
a_{i}(k) & =p p_{i i} \sum_{n=0}^{k-1} h_{i i}^{X}(k-1-n)\left[\theta f_{i i}(n)+(1-\theta) \sum_{k=1}^{2} p_{i k} f_{i k}(n)\right], \quad k=2,3, \ldots, i=1,2, \\
b_{i j} & =v p q(1-\theta) \sum_{k=1}^{2} p_{i k} q_{i k}(1) g_{i k}(1) p_{k j} q_{k j}(1) f_{k j}(1), \quad i, j=1,2, \\
f_{k} & =\sum_{n=0}^{k}\left[a_{1}(n) a_{2}(k-n)-e_{12}(n) e_{21}(k-n)\right], \\
g_{k}^{(1)} & =\sum_{n=0}^{k} W_{1}(n) f_{k-n}, \\
h_{k}^{(1)} & =e_{1}(0) a_{2}(k)+e_{1}(1) a_{2}(k-1)-e_{2}(0) e_{12}(k)-e_{2}(1) e_{12}(k-1), \quad k \in \mathbb{N},
\end{aligned}
$$

where $e_{i}(k)$ and $e_{i j}(k)$ are the coefficients of $s^{k}$ on functions $\tilde{e}_{i}(s)$ and $\tilde{e}_{i j}(s)$. In addition, $a_{i}(-1)=0, e_{i j}$ $(-1)=0, i, j=1,2$.

Comparing the coefficients of $s^{k}$ in both sides of (20), we get $g_{k}^{(1)}=h_{k}^{(1)}, k \in \mathbb{N}$. So we have

$$
\sum_{n=0}^{k} W_{1}(n) f_{k-n}=h_{k}^{(1)}, \quad k \in \mathbb{N} \text {. }
$$

In a similar way, we obtain that

$$
\sum_{n=0}^{k} W_{2}(n) f_{k-n}=h_{k}^{(2)}, \quad k \in \mathbb{N}
$$

where $\quad h_{k}^{(2)}=-e_{1}(0) e_{21}(k)-e_{1}(1) e_{21}(k-1)+e_{2}(0) a_{1}(k)$ $+e_{2}(1) a_{1}(k-1)$. 
From (22) and (23), we know that $f_{k}$ play a decisive role in the value of $\left\{W_{i}(k), k \in \mathbb{N}, i=1,2\right\}$. So we discuss the values of $f_{k}$ in the following lemma.

Lemma 1. If $f_{0}=0$, then $f_{1} \neq 0$.

Proof. Note that

$$
\begin{aligned}
f_{0}= & e_{11}(0) e_{22}(0)-e_{12}(0) e_{21}(0), \\
f_{1}= & e_{11}(0)\left[e_{22}(1)-\frac{1}{v}\right]+e_{22}(0)\left[e_{11}(1)-\frac{1}{v}\right] \\
& -e_{21}(0) e_{12}(1)-e_{12}(0) e_{21}(1) .
\end{aligned}
$$

If $f_{0}=0$, we can derive $f_{1} \neq 0$ by using reduction to absurdity. Assuming $f_{1}=0$ yields

$$
\begin{aligned}
e_{11}(0) & =0, \\
e_{22}(0) & =0, \\
e_{21}(0) e_{12}(1) & =0, \\
e_{21}(1) e_{12}(0) & =0,
\end{aligned}
$$

where $e_{11}(0)=q p_{11} q_{11}(1)$ means $q_{11}(1)=0$, and $e_{22}(0)=$ $q p_{22} q_{22}(1)$ means $q_{22}(1)=0$.

Since $f_{0}=0$, we get $e_{21}(0) e_{12}(0)=0$. Note that $\sum_{i=1}^{2} \sum_{j=1}^{2} q_{i j}(1) \neq 0$, there are only two situations: (i) If $e_{12}(0)=0$ and $e_{21}(0) \neq 0$, we have

$$
\begin{aligned}
e_{12}(1)= & q p_{12} q_{12}(0)+p p_{12} h_{12}^{X}(0) \\
& \cdot\left[\theta f_{12}(0)+(1-\theta) \sum_{k=1}^{2} p_{12} f_{12}(0)\right]=0,
\end{aligned}
$$

which means $q_{12}(0)=0$, and $e_{12}(0)=0$ yields $q_{12}(1)=0$. The result $\sum_{l=0}^{1} q_{12}(l) \neq 1$ is obviously not in agreement with reality, so there is no such situation.

(ii) If $e_{21}(0)=0$ and $e_{12}(0) \neq 0$, we can derive in a similar way that $\sum_{l=0}^{1} q_{21}(l) \neq 1$, which is also not realistic. The proof is complete.

In order to obtain the explicit expressions of $V_{i}(u, b)$, we shall distinguish two cases.

(1) If $f_{0} \neq 0$, equations (22) and (23) yield

$$
W_{i}(k)=\frac{1}{f_{0}}\left[h_{k}^{(i)}-\sum_{n=0}^{k-1} W_{i}(n) f_{k-n}\right], \quad i=1,2, k \in \mathbb{N}_{+} .
$$

\section{Denote}

$$
\begin{aligned}
& \xi_{k}^{(1)}=e_{11}(0) a_{2}(k)-b_{11} a_{2}(k-1)-e_{21}(0) e_{12}(k)+b_{21} e_{12}(k-1), \\
& \eta_{k}^{(1)}=e_{12}(0) a_{2}(k)-b_{12} a_{2}(k-1)-e_{22}(0) e_{12}(k)+b_{22} e_{12}(k-1), \\
& \xi_{k}^{(2)}=e_{21}(0) a_{1}(k)-b_{21} a_{1}(k-1)-e_{11}(0) e_{21}(k)+b_{11} e_{21}(k-1), \\
& \eta_{k}^{(2)}=e_{22}(0) a_{1}(k)-b_{22} a_{1}(k-1)-e_{12}(0) e_{21}(k)+b_{12} e_{21}(k-1) .
\end{aligned}
$$

Note that $h_{k}^{(i)}$ can be rewritten as

$$
h_{k}^{(i)}=\xi_{k}^{(i)} W_{1}(0)+\eta_{k}^{(i)} W_{2}(0), \quad i=1,2, k \in \mathbb{N},
$$

which are determined by $W_{1}(0)$ and $W_{2}(0)$, and we can see from (27) that $\left\{W_{1}(k), W_{2}(k)\right\}_{k \in \mathbb{N}_{+}}$is also determined by the initial values $W_{1}(0)$ and $W_{2}(0)$. Therefore, apart from a multiplicative constant, the solution of (15) and (16) is unique. For $j=1,2$, we set $\left\{C_{1 j}(k), C_{2 j}(k)\right\}$ to be the linearly independent particular solutions of (15) and (16) with initial conditions $C_{i j}(0)=I_{(i=j)}$. Then the general solution of (15) and (16) is given by

$$
W_{i}(u)=\sum_{j=1}^{2} W_{j}(0) C_{i j}(u), \quad u \in \mathbb{N}, i=1,2 .
$$

The same procedure may be easily adapted to obtain the solutions of (13) and (14), that is,

$$
V_{i}(u, b)=\sum_{j=1}^{2} V_{j}(0, b) C_{i j}(u), \quad i=1,2, u=0,1, \ldots, b+1 .
$$

Let

$$
\begin{aligned}
\overrightarrow{\mathbf{V}}(u, b) & =\left(V_{1}(u, b), V_{2}(u, b)\right)^{T}, \\
\mathbf{C}(u) & =\left(C_{i j}(u)\right)_{i, j=1,2^{2}} \\
\overrightarrow{1} & =(1,1)^{T} .
\end{aligned}
$$

Rewriting (31) into matrix form as follows:

$\overrightarrow{\mathbf{V}}(u, b)=\mathbf{C}(u) \overrightarrow{\mathbf{V}}(0, b), \quad i=1,2, u=0,1, \ldots, b+1$.

Combining (33) with the boundary condition $\overrightarrow{\mathbf{V}}(b+1, b)=\overrightarrow{1}+\overrightarrow{\mathbf{V}}(b, b)$, we get 


$$
\overrightarrow{\mathbf{V}}(0, b)=[\mathbf{C}(b+1)-\mathbf{C}(b)]^{-1} \overrightarrow{1},
$$

which in turn yields that

$$
\overrightarrow{\mathbf{V}}(u, b)=\mathbf{C}(u)[\mathbf{C}(b+1)-\mathbf{C}(b)]^{-1} \overrightarrow{1}, \quad u=0,1, \ldots, b .
$$

(2) If $f_{0}=0$, Lemma 1 guarantees that $f_{1} \neq 0$. Based on (22) and (23), we get $W_{i}(0)=\left(1 / f_{1}\right) h_{1}^{(i)}, i=1,2$, and

$$
W_{i}(k)=\frac{1}{f_{1}}\left[h_{k+1}^{(i)}-\sum_{n=0}^{k-1} W_{i}(n) f_{k+1-n}\right], \quad i=1,2, k \in \mathbb{N}_{+} .
$$

Define

$$
\begin{aligned}
\mathbf{J}_{1} & =\left(\begin{array}{cc}
\xi_{1}^{(1)}-f_{1} & \eta_{1}^{(1)} \\
\xi_{1}^{(2)} & \eta_{1}^{(2)}-f_{1}
\end{array}\right), \\
\overrightarrow{\mathbf{W}}(0) & =\left(W_{1}(0), W_{2}(0)\right)^{T}, \\
\overrightarrow{0} & =(0,0)^{T} .
\end{aligned}
$$

We obtain that

$$
\mathbf{J}_{1} \overrightarrow{\mathbf{W}}(0)=\overrightarrow{0}
$$

Let

$$
\begin{aligned}
\mathbf{D}_{0} & =\left(\begin{array}{cc}
e_{22}(0) & -e_{12}(0) \\
-e_{21}(0) & e_{11}(0)
\end{array}\right), \\
\mathbf{E} & =\left(\begin{array}{cc}
\frac{1}{v}-e_{11}(1) & -e_{12}(1) \\
-e_{21}(1) & \frac{1}{v}-e_{22}(1)
\end{array}\right) .
\end{aligned}
$$

Because of $\mathbf{J}_{1}=\mathbf{D}_{0} \mathbf{E}, \mathbf{D}_{0} \neq 0,\left|\mathbf{D}_{0}\right|=f_{0}=0$ and $|\mathbf{E}|>0$, we have $\operatorname{rank}\left(\mathbf{J}_{1}\right)=\operatorname{rank}\left(\mathbf{D}_{0}\right)=1$. Equation (38) shows that only one of $W_{1}(0)$ and $W_{2}(0)$ is free variable. Assuming that $W_{1}(0)$ is the free variable without loss of generality, then we know that $\left\{W_{1}(k), W_{2}(k)\right\}_{k \in \mathbb{N}_{+}}$is determined by the initial value $W_{1}(0)$. Set $\left\{W_{1}^{(1)}(u), W_{2}^{(1)}(u), u \in \mathbb{N}\right\}$ to be the linearly independent particular solution of (15) and (16) with initial conditions $W_{1}^{(1)}(0)=1$, and then the general solution of (15) and (16) is of the form:

$$
\left(W_{1}(u), W_{2}(u)\right)=W_{1}(0)\left(W_{1}^{(1)}(u), W_{2}^{(1)}(u)\right), \quad u \in \mathbb{N} .
$$

Therefore, the solution of (13) and (14) is given by

$$
\begin{aligned}
\left(V_{1}(u, b), V_{2}(u, b)\right) & =V_{1}(0, b)\left(W_{1}^{(1)}(u), W_{2}^{(1)}(u)\right), \\
u & =0,1, \ldots, b+1 .
\end{aligned}
$$

Combining the above equation with $V_{1}(b+1, b)=1+$ $V_{1}(b, b)$ gives

$$
V_{1}(0, b)=\left[W_{1}^{(1)}(b+1)-W_{1}^{(1)}(b)\right]^{-1} .
$$

So we have

$$
\begin{aligned}
\left(V_{1}(u, b), V_{2}(u, b)\right)= & {\left[W_{1}^{(1)}(b+1)-W_{1}^{(1)}(b)\right]^{-1} } \\
& \cdot\left(W_{1}^{(1)}(u), W_{2}^{(1)}(u)\right), \\
u= & 0,1, \ldots, b .
\end{aligned}
$$

To sum up, the main result of this paper is as follows.

Theorem 1. For any given dividend barrier $b \in \mathbb{N}_{+}$and initial surplus $u=0,1, \ldots, b$, the total expected discounted dividends until ruin satisfies the following explicit expression:

$$
\overrightarrow{\mathbf{V}}(u, b)= \begin{cases}\mathbf{C}(u)[\mathbf{C}(b+1)-\mathbf{C}(b)]^{-1} \overrightarrow{1}, & f_{0} \neq 0 \\ {\left[W_{1}^{(1)}(b+1)-W_{1}^{(1)}(b)\right]^{-1}\left(W_{1}^{(1)}(u), W_{2}^{(1)}(u)\right),} & f_{0}=0\end{cases}
$$

where $\mathbf{C}(u)=\left(C_{i j}(u)\right)_{i, j=1,2}$ with $C_{i j}(0)=I_{(i=j)}$ and $C_{i j}$ $(k)=\left(1 / f_{0}\right)\left[\xi_{k}^{(i)} I_{\{j=1\}}+\eta_{k}^{(i)} I_{\{j=2\}}-\sum_{n=0}^{k-1} C_{i j}(n) f_{k-n}\right], 2$ $k \in \mathbb{N}_{+}, i, j=1,2 ; \quad W_{1}^{(1)}(0)=1, W_{2}^{(1)}(0)=0, W_{i}^{(1)}(k)=$ $\left(1 / f_{1}\right)\left[\xi_{k+1}^{(i)}-\sum_{n=0}^{k-1} W_{i}^{(1)}(n) f_{k+1-n}\right], k \in \mathbb{N}_{+}, i=1,2$.

\section{Numerical Simulation}

In this section, we use several examples to illustrate the conclusions of $f_{0} \neq 0$ in Section 3 and explain the impact of $u, b, v, p$, and $\theta$ on the total expected discounted dividends. In the following numerical examples, the transition 
TABLE 1: The distribution of premium income.

\begin{tabular}{ccccc}
\hline$l$ & $q_{11}(l)$ & $q_{12}(l)$ & $q_{21}(l)$ & \\
\hline 0 & $1 / 10$ & $2 / 9$ & $1 / 6$ & 0 \\
1 & $9 / 10$ & $7 / 9$ & $5 / 6$ & 1 \\
\hline
\end{tabular}

TABLE 2: The distribution of main claim.

\begin{tabular}{lcccc}
\hline$n$ & $g_{11}(n)$ & $g_{12}(n)$ & $g_{21}(n)$ & $g_{22}(n)$ \\
\hline 1 & $13 / 16$ & $3 / 4$ & $5 / 6$ & 1 \\
2 & $1 / 8$ & $1 / 4$ & 0 & 0 \\
3 & $1 / 16$ & 0 & $1 / 6$ & 0 \\
$\geq 4$ & 0 & 0 & 0 & 0 \\
\hline
\end{tabular}

TABLE 3: Values of $V_{i}(u, b)$ when $v=0.93$

\begin{tabular}{|c|c|c|c|c|c|c|c|c|c|c|}
\hline & $i$ & $b=0$ & $b=1$ & $b=2$ & $b=3$ & $b=4$ & $b=5$ & $b=6$ & $b=7$ & $b=8$ \\
\hline \multirow{2}{*}{$u=0$} & 1 & 2.7495 & 2.6384 & 2.4991 & 2.2867 & 2.0104 & 1.7367 & 1.4871 & 1.2678 & 1.0786 \\
\hline & 2 & 3.0406 & 2.9996 & 2.8248 & 2.5835 & 2.2715 & 1.9625 & 1.6805 & 1.4327 & 1.2188 \\
\hline \multirow{2}{*}{$u=1$} & 1 & 3.7495 & 3.5290 & 3.3566 & 3.0723 & 2.7009 & 2.3331 & 1.9977 & 1.7031 & 1.4488 \\
\hline & 2 & 4.0406 & 4.0274 & 3.7846 & 3.4606 & 3.0428 & 2.6290 & 2.2513 & 1.9193 & 1.6328 \\
\hline \multirow{2}{*}{$u=2$} & 1 & 4.7495 & 4.5290 & 4.3473 & 3.9821 & 3.5001 & 3.0231 & 2.5884 & 2.2066 & 1.8771 \\
\hline & 2 & 5.0406 & 5.0274 & 4.7007 & 4.2965 & 3.7781 & 3.2645 & 2.7956 & 2.3835 & 2.0277 \\
\hline \multirow{2}{*}{$u=3$} & 1 & 5.7495 & 5.5290 & 5.3473 & 4.9066 & 4.3112 & 3.7225 & 3.1867 & 2.7164 & 2.3107 \\
\hline & 2 & 6.0406 & 6.0274 & 5.7007 & 5.2055 & 4.5783 & 3.9566 & 3.3887 & 2.8892 & 2.4580 \\
\hline \multirow{2}{*}{$u=4$} & 1 & 6.7495 & 6.5290 & 6.3473 & 5.9066 & 5.1856 & 4.4744 & 3.8288 & 3.2631 & 2.7756 \\
\hline & 2 & 7.0406 & 7.0274 & 6.7007 & 6.2055 & 5.4603 & 4.7208 & 4.0440 & 3.4483 & 2.9337 \\
\hline \multirow{2}{*}{$u=5$} & 1 & 7.7495 & 7.5290 & 7.3473 & 6.9066 & 6.1856 & 5.3283 & 4.5553 & 3.8807 & 3.3003 \\
\hline & 2 & 8.0406 & 8.0274 & 7.7007 & 7.2055 & 6.4603 & 5.5906 & 4.7916 & 4.0867 & 3.4773 \\
\hline
\end{tabular}

TABLE 4: Values of $V_{i}(u, b)$ when $v=0.95$

\begin{tabular}{|c|c|c|c|c|c|c|c|c|c|c|}
\hline & $i$ & $b=0$ & $b=1$ & $b=2$ & $b=3$ & $b=4$ & $b=5$ & $b=6$ & $b=7$ & $b=8$ \\
\hline \multirow{2}{*}{$u=0$} & 1 & 3.0651 & 3.1731 & 3.2602 & 3.2280 & 3.0292 & 2.7687 & 2.4935 & 2.2277 & 1.9819 \\
\hline & 2 & 3.3921 & 3.6103 & 3.6819 & 3.6419 & 3.4173 & 3.1235 & 2.8130 & 2.5132 & 2.2360 \\
\hline \multirow{2}{*}{$u=1$} & 1 & 4.0651 & 4.1265 & 4.2627 & 4.2236 & 3.9637 & 3.6227 & 3.2625 & 2.9148 & 2.5932 \\
\hline & 2 & 4.3921 & 4.7233 & 4.8039 & 4.7500 & 4.4569 & 4.0736 & 3.6688 & 3.2778 & 2.9162 \\
\hline \multirow{2}{*}{$u=2$} & 1 & 5.0651 & 5.1265 & 5.3592 & 5.3184 & 4.9918 & 4.5622 & 4.1086 & 3.6706 & 3.2656 \\
\hline & 2 & 5.3921 & 5.7233 & 5.7831 & 5.7131 & 5.3603 & 4.8994 & 4.4126 & 3.9424 & 3.5075 \\
\hline \multirow{2}{*}{$u=3$} & 1 & 6.0651 & 6.1265 & 6.3592 & 6.3342 & 5.9470 & 5.4349 & 4.8941 & 4.3722 & 3.8898 \\
\hline & 2 & 6.3921 & 6.7233 & 6.7831 & 6.6872 & 6.2730 & 5.7339 & 5.1644 & 4.6142 & 4.1052 \\
\hline \multirow{2}{*}{$u=4$} & 1 & 7.0651 & 7.1265 & 7.3592 & 7.3342 & 6.8909 & 6.2966 & 5.6691 & 5.0641 & 4.5051 \\
\hline & 2 & 7.3921 & 7.7233 & 7.7831 & 7.6872 & 7.2082 & 6.5892 & 5.9353 & 5.3032 & 4.7183 \\
\hline \multirow{2}{*}{$u=5$} & 1 & 8.0651 & 8.1265 & 8.3592 & 8.3342 & 7.8909 & 7.2077 & 6.4869 & 5.7934 & 5.1534 \\
\hline & 2 & 8.3921 & 8.7233 & 8.7831 & 8.6872 & 8.2082 & 7.5049 & 6.7616 & 6.0422 & 5.3762 \\
\hline
\end{tabular}

probability matrix of Markov chain is set to be $\left(\begin{array}{ll}0.7 & 0.3 \\ 0.2 & 0.8\end{array}\right)$, the distribution of premium income is shown in Table 1, the distribution of main claim is shown in Table 2, and we assume that the distribution of by-claim is the same as main claim.

Example 1. In this example, we set $p=0.25$ and $\theta=0.3$. The results of $V_{i}(u, b)$ for $v=0.93,0.95,0.97$ are listed in Tables 3-5, respectively. We can see that the expected discounted dividends are gradually increasing with the increase of the initial surplus $u$ or one-period discount factor $v$. Given the initial surplus $u$, set the optimal dividend boundary $b^{*}$ be the boundary value which maximizes the expected discounted dividends $V_{i}(u, b)$. From Table 3 , it is easy to see that $b^{*}=0$ when $v=0.93$. Tables 4 and 5 show that $b^{*}=2$ when $v=0.95$ and $b^{*}=4$ when $v=0.97$. The results in this example imply that it would be better to pay dividends earlier when the discount factor $v$ is smaller.

Example 2. In this example, we take $v=0.95, b=8$, and $\theta=0.3$ and investigate the effect of $p$ on the expected discounted dividends $V_{i}(u, b)$. It can be seen from Table 6 that the expected discounted dividends are a decreasing function of $p$, and the result is in accordance with the fact. 
TABLE 5: Values of $V_{i}(u, b)$ when $v=0.97$

\begin{tabular}{lcccccccccc}
\hline & $i$ & $b=0$ & $b=1$ & $b=2$ & $b=3$ & $b=4$ & $b=5$ & $b=6$ & $b=7$ & $b=8$ \\
\hline \multirow{2}{*}{$u=0$} & 1 & 3.4458 & 3.9036 & 4.4567 & 4.9317 & 5.0810 & 5.0235 & 4.8360 & 4.5801 & 4.2966 \\
& 2 & 3.8163 & 4.4453 & 5.0289 & 5.5564 & 5.7232 & 5.6582 & 5.4469 & 5.1587 & 4.8394 \\
$u=1$ & 1 & 4.4458 & 4.9370 & 5.6744 & 6.2860 & 6.4775 & 6.4044 & 6.1654 & 5.8391 & 5.4777 \\
& 2 & 4.8163 & 5.6693 & 6.3918 & 7.0582 & 7.2693 & 7.1866 & 6.9183 & 6.5522 & 6.1466 \\
$u=2$ & 1 & 5.4458 & 5.9370 & 6.9269 & 7.6922 & 7.9298 & 7.8409 & 7.5483 & 7.1489 & 6.7064 \\
& 2 & 5.8163 & 6.6693 & 7.4579 & 8.2241 & 8.4681 & 8.3714 & 8.0589 & 7.6324 & 7.1600 \\
$u=3$ & 1 & 6.4458 & 6.9370 & 7.9269 & 8.8545 & 9.1369 & 9.0361 & 8.6991 & 8.2386 & 7.7287 \\
& 2 & 6.8163 & 7.6693 & 8.4579 & 9.2962 & 9.5667 & 9.4566 & 9.1034 & 8.6217 & 8.0881 \\
$u=4$ & 1 & 7.4458 & 7.9370 & 8.9269 & 9.8545 & 10.1930 & 10.0848 & 9.7090 & 9.1950 & 8.6258 \\
& 2 & 7.8163 & 8.6693 & 9.4579 & 10.2962 & 10.5815 & 10.4572 & 10.0664 & 9.5338 & 8.9438 \\
$u=5$ & 1 & 8.4458 & 8.9370 & 9.9269 & 10.8545 & 11.1930 & 11.0854 & 10.6735 & 10.1082 & 9.4821 \\
& 2 & 8.8163 & 9.6693 & 10.4579 & 11.2962 & 11.5815 & 11.4388 & 11.0106 & 10.4282 & 9.7831 \\
\hline
\end{tabular}

TABle 6: Values of $V_{i}(u, b)$ when $v=0.95, b=8$, and $\theta=0.3$.

\begin{tabular}{|c|c|c|c|c|c|c|c|c|c|}
\hline & $i$ & $p=0.05$ & $p=0.10$ & $p=0.15$ & $p=0.20$ & $p=0.25$ & $p=0.30$ & $p=0.40$ & $p=0.50$ \\
\hline \multirow{2}{*}{$u=0$} & 1 & 8.8392 & 6.6890 & 4.8048 & 3.2247 & 1.9819 & 1.0907 & 0.2202 & 0.0250 \\
\hline & 2 & 9.1448 & 7.0774 & 5.1970 & 3.5635 & 2.2360 & 1.2551 & 0.2627 & 0.0307 \\
\hline \multirow{2}{*}{$u=1$} & 1 & 9.6611 & 7.5912 & 5.6875 & 4.0016 & 2.5932 & 1.5145 & 0.3523 & 0.0479 \\
\hline & 2 & 9.9655 & 7.9947 & 6.1201 & 4.4017 & 2.9162 & 1.7408 & 0.4222 & 0.0595 \\
\hline \multirow{2}{*}{$u=2$} & 1 & 10.4853 & 8.4901 & 6.5824 & 4.8166 & 3.2656 & 2.0094 & 0.5327 & 0.0863 \\
\hline & 2 & 10.6805 & 8.7312 & 6.8512 & 5.0866 & 3.5075 & 2.1997 & 0.6087 & 0.1030 \\
\hline \multirow[b]{2}{*}{$u=3$} & 1 & 11.2369 & 9.2628 & 7.3470 & 5.5332 & 3.8898 & 2.5039 & 0.7512 & 0.1456 \\
\hline & 2 & 11.4095 & 9.4604 & 7.5651 & 5.7595 & 4.1052 & 2.6877 & 0.8413 & 0.1712 \\
\hline \multirow{2}{*}{$u=4$} & 1 & 11.9809 & 9.9978 & 8.0648 & 6.2146 & 4.5051 & 3.0202 & 1.0216 & 0.2383 \\
\hline & 2 & 12.1553 & 10.1900 & 8.2728 & 6.4317 & 4.7183 & 3.2124 & 1.1328 & 0.2781 \\
\hline \multirow{2}{*}{$u=5$} & 1 & 12.7585 & 10.7568 & 8.8011 & 6.9170 & 5.1534 & 3.5870 & 1.3640 & 0.3842 \\
\hline & 2 & 12.9415 & 10.9558 & 9.0143 & 7.1396 & 5.3762 & 3.7960 & 1.5035 & 0.4462 \\
\hline
\end{tabular}

TABle 7: Values of $V_{i}(u, b)$ when $v=0.95, b=8$, and $p=0.25$.

\begin{tabular}{|c|c|c|c|c|c|c|c|}
\hline & $i$ & $\theta=0$ & $\theta=0.2$ & $\theta=0.4$ & $\theta=0.6$ & $\theta=0.8$ & $\theta=1$ \\
\hline \multirow{2}{*}{$u=0$} & 1 & 2.0822 & 2.0141 & 1.9509 & 1.8920 & 1.8370 & 1.7855 \\
\hline & 2 & 2.3755 & 2.2807 & 2.1930 & 2.1117 & 2.0361 & 1.9656 \\
\hline \multirow{2}{*}{$u=1$} & 1 & 2.6327 & 2.6059 & 2.5810 & 2.5578 & 2.5361 & 2.5158 \\
\hline & 2 & 2.9512 & 2.9274 & 2.9055 & 2.8854 & 2.8669 & 2.8499 \\
\hline \multirow{2}{*}{$u=2$} & 1 & 3.2963 & 3.2755 & 3.2559 & 3.2374 & 3.2197 & 3.2029 \\
\hline & 2 & 3.5201 & 3.5114 & 3.5038 & 3.4971 & 3.4914 & 3.4864 \\
\hline \multirow{2}{*}{$u=3$} & 1 & 3.9092 & 3.8961 & 3.8836 & 3.8715 & 3.8599 & 3.8487 \\
\hline & 2 & 4.1132 & 4.1077 & 4.1029 & 4.0989 & 4.0955 & 4.0927 \\
\hline \multirow{2}{*}{$u=4$} & 1 & 4.5186 & 4.5095 & 4.5008 & 4.4924 & 4.4844 & 4.4767 \\
\hline & 2 & 4.7226 & 4.7196 & 4.7172 & 4.7150 & 4.7133 & 4.7119 \\
\hline \multirow{2}{*}{$u=5$} & 1 & 5.1637 & 5.1567 & 5.1500 & 5.1436 & 5.1374 & 5.1313 \\
\hline & 2 & 5.3776 & 5.3766 & 5.3758 & 5.3753 & 5.3749 & 5.3747 \\
\hline
\end{tabular}

Example 3. In this example, we discuss the impact of $\theta$ on the expected discounted dividends $V_{i}(u, b)$. Let $v=0.95$, $b=8$, and $p=0.25$, The values of $V_{i}(u, b)$ are shown in Table 7. It is easy to see that the expected discounted dividends $V_{i}(u, b)$ gradually decrease with the increase of $\theta$, and the effect of $\theta$ on $V_{i}(u, b)$ is decreasing with respect to the initial surplus $u$.

\section{Data Availability}

No data were used to support this study.

\section{Conflicts of Interest}

The authors declare that they have no conflicts of interest.

\section{Acknowledgments}

This work was supported by the National Natural Science Foundation of China (nos. 11701088 and 11701087), the Natural Science Foundation of Fujian Province (nos. 2018J05003 and 2019J01673), the Shandong Provincial Natural Science Foundation (no. ZR2018MG002), the 
Program for Innovative Research Team in Science and Technology in Fujian Province University, and the grant "Probability and Statistics: Theory and Application (no. IRTL1704)" from Fujian Normal University.

\section{References}

[1] B. De Finetti, "Su un'impostazione alternativa della teoria collettiva del rischio," Transactions of the XVth International Congress of Actuaries, vol. 2, pp. 433-443, 1957.

[2] M. M. Claramunt, M. Marmol, and A. Alegre, "A note on the expected present value of dividends with a constant barrier in the discrete time model," Bulletin of the Swiss Association of Actuaries, vol. 2, pp. 149-159, 2003.

[3] X. Zhou, "On a classical risk model with a constant dividend barrier," North American Actuarial Journal, vol. 9, no. 4, pp. 95-108, 2005.

[4] D. Landriault, "Constant dividend barrier in a risk model with interclaim-dependent claim sizes," Insurance: Mathematics and Economics, vol. 42, no. 1, pp. 31-38, 2008.

[5] H. U. Gerber, E. S. W. Shiu, and H. Yang, "An elementary approach to discrete models of dividend strategies," Insurance: Mathematics and Economics, vol. 46, no. 1, pp. 109-116, 2010.

[6] M. Chen, X. Peng, and J. Guo, "Optimal dividend problem with a nonlinear regular-singular stochastic control," Insurance: Mathematics and Economics, vol. 52, no. 3, pp. 448-456, 2013.

[7] M. Chen and K. C. Yuen, "Optimal dividend and reinsurance in the presence of two reinsurers," Journal of Applied Probability, vol. 53, no. 2, pp. 554-571, 2016.

[8] X. Peng, L. Bai, and J. Guo, "Optimal control with restrictions for a diffusion risk model under constant interest force," Applied Mathematics \& Optimization, vol. 73, no. 1, pp. 115-136, 2016.

[9] X. Peng, W. Su, and Z. Zhang, "On a perturbed compound Poisson risk model under a periodic threshold-type dividend strategy," Journal of Industrial \& Management Optimization, vol. 16, no. 4, pp. 1967-1986, 2020.

[10] K. C. Yuen and J. Y. Guo, "Ruin probabilities for time-correlated claims in the compound binomial model," Insurance: Mathematics and Economics, vol. 29, no. 1, pp. 47-57, 2001.

[11] J.-Z. Li and R. Wu, "The Gerber-Shiu discounted penalty function for a compound binomial risk model with byclaims," Acta Mathematicae Applicatae Sinica, English Series, vol. 31, no. 1, pp. 181-190, 2015.

[12] Y. Xiao and J. Guo, "The compound binomial risk model with time-correlated claims," Insurance: Mathematics and Economics, vol. 41, no. 1, pp. 124-133, 2007.

[13] Z. H. Bao and H. Liu, "The compound binomial risk model with delayed claims and random income," Mathematical and Computer Modelling, vol. 55, no. 3-4, pp. 1315-1323, 2012.

[14] J. Zhou, X. Mo, H. Ou, and X. Yang, "Expected present value of total dividends in the compound binomial model with delayed claims and random income," Acta Mathematica Scientia, vol. 33, no. 6, pp. 1639-1651, 2013.

[15] K. C. Yuen, J. Li, and R. Wu, "On a discrete-time risk model with delayed claims and dividends," Risk and Decision Analysis, vol. 4, no. 1, pp. 3-16, 2013.

[16] C. L. Liu and Z. M. Zhang, "On a discrete risk model with delayed claims and a randomized dividend strategy," Advances in Difference Equations, vol. 2015, no. 1, pp. 1-14, Article ID 284, 2015.
[17] Y. Deng, J. Liu, Y. Huang, M. Li, and J. Zhou, "On a discrete interaction risk model with delayed claims and stochastic incomes under random discount rates," Communications in Statistics-Theory and Methods, vol. 47, no. 23, pp. 58675883, 2018.

[18] A. V. Boikov, "The Cramer-Lundberg model with stochastic premium process," Theory of Probability \& Its Applications, vol. 47, no. 3, pp. 489-493, 2003.

[19] Z.-H. Bao, "The expected discounted penalty at ruin in the risk process with random income," Applied Mathematics and Computation, vol. 179, no. 2, pp. 559-566, 2006.

[20] W. Zou, J.-W. Gao, and J.-H. Xie, "On the expected discounted penalty function and optimal dividend strategy for a risk model with random incomes and interclaim-dependent claim sizes," Journal of Computational and Applied Mathematics, vol. 255, pp. 270-281, 2014.

[21] H. Yang and Z. Zhang, "On a class of renewal risk model with random income," Applied Stochastic Models in Business and Industry, vol. 25, no. 6, pp. 678-695, 2009.

[22] Z. Zhang and H. Yang, "On a risk model with stochastic premiums income and dependence between income and loss," Journal of Computational and Applied Mathematics, vol. 234, no. 1, pp. 44-57, 2010.

[23] M. Jacobsen, "The time to ruin in some additive risk models with random premium rates," Journal of Applied Probability, vol. 49, no. 4, pp. 915-938, 2012.

[24] W. G. Yu, "Randomized dividends in a discrete insurance risk model with stochastic premium income," Mathematical Problems in Engineering, vol. 2013, Article ID 579534, 9 pages, 2013.

[25] J. Zhu and H. Yang, "Ruin theory for a Markov regimeswitching model under a threshold dividend strategy," Insurance: Mathematics and Economics, vol. 42, no. 1, pp. 311-318, 2008.

[26] M. Chen, K. C. Yuen, and J. Guo, "Survival probabilities in a discrete semi-Markov risk model," Applied Mathematics and Computation, vol. 232, pp. 205-215, 2014.

[27] K. C. Yuen, M. Chen, and K. P. Wat, "On the expected penalty functions in a discrete semi-Markov risk model with randomized dividends," Journal of Computational and Applied Mathematics, vol. 311, pp. 239-251, 2017.

[28] Y. Lu and S. M. Li, "On the severity of ruin in a Markovmodulated risk model," Scandinavian Actuarial Journal, vol. 2006, no. 4, pp. 183-202, 2006.

[29] P. Diko and M. Usábel, "A numerical method for the expected penalty-reward function in a Markov-modulated jump-diffusion process," Insurance: Mathematics and Economics, vol. 49, no. 1, pp. 126-131, 2011.

[30] M. Chen, J. Guo, and X. Wu, "Expected discounted dividends in a discrete semi-Markov risk model," Journal of Computational and Applied Mathematics, vol. 266, no. 2, pp. 1-17, 2014.

[31] H. Huo, X. Zou, and X. Guo, "The risk probability criterion for discounted continuous-time Markov decision processes," Discrete Event Dynamic Systems, vol. 27, no. 4, pp. 675-699, 2017. 